\title{
Les deux piliers de la construction territoriale : coordination des acteurs et ressources territoriales
}

\section{Hadjou Lamara}

\section{(2) OpenEdition}

\section{Journals}

\section{Édition électronique}

URL : http://journals.openedition.org/developpementdurable/8208

DOI : 10.4000/developpementdurable.8208

ISSN : 1772-9971

Éditeur

Association DD\&T

\section{Référence électronique}

Hadjou Lamara, «Les deux piliers de la construction territoriale : coordination des acteurs et ressources territoriales », Développement durable et territoires [En ligne], Varia (2004-2010), mis en ligne le 07 juillet 2009, consulté le 30 avril 2019. URL : http://journals.openedition.org/ developpementdurable/8208; DOI : 10.4000/developpementdurable.8208

Ce document a été généré automatiquement le 30 avril 2019.

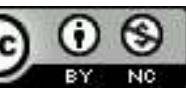

Développement Durable et Territoires est mis à disposition selon les termes de la licence Creative Commons Attribution - Pas d'Utilisation Commerciale 4.0 International. 


\title{
Les deux piliers de la construction territoriale : coordination des acteurs et ressources territoriales
}

\author{
Hadjou Lamara
}

1 C'est à partir des travaux de l'initiateur de la théorie des milieux innovateurs, Philippe Aydalot (Camagni et Maillat, 2006; Tabariés, 2005 ; Peyrache, 1999), que la variable territoriale commence à s'imposer comme le nouveau paradigme du développement. Celui-ci en se retournant contre les théories classiques et néoclassiques de l'économie, tente de remettre en cause la conception très réductrice de la dimension spatiale $\mathrm{du}$ développement. Après le recensement de 1982 en France, Philippe Aydalot note avec perspicacité un "retournement des hiérarchies spatiales». En clair, les espaces à forte composante industrielle plongent avec la crise du fordisme, alors que de nouveaux espaces, sans tradition industrielle particulière émergent et démontrent une forte résistance à la concurrence mondiale qui devient de plus en plus pressante (Lipietz, 1995). Le succès de ces espaces est "essentiellement dû à leur dynamique interne » (Lipietz, 1995, p. 3) ; le territoire est donc autoproduit par une régulation locale instituée par les acteurs. A la suite de cette première intuition, X. Greffe (1984), selon R. Kahn (2007) fait un pas supplémentaire en abandonnant l'hypothèse d'homogénéité spatiale. X. Greffe (1984) affirme que les espaces locaux déployant une stratégie de développement sur une assise spatiale doivent désormais porter le qualificatif de territoire.

2 L'espace, selon G. Benko (1996), n'est pas seulement un cadre de la localisation des agents économiques, c'est "aussi le cadre de l'émergence d'un acteur économique particulier dont l'importance aujourd'hui est abondamment soulignée: le Territoire»(p. 7). S'inspirant des intuitions d'Alfred Marshall réinterprétées sous le filtre des théories institutionnalistes des coûts de transaction (Coase-Williamson-North) et évolutionnistes du changement technique (Nelson\&Winter), les premiers travaux de recherche sur le territoire sont menés conjointement en Italie sur les districts (Becattini, Bagnasco et Trigilia), en France sur les systèmes productifs localisés (Courlet et Pecqueur) et sur les milieux innovateurs 
(GREMI), et aux Etats-Unis par l'école californienne de géographie économique sur les métropoles (Scott-Storper-Walker). Le territoire s'impose ainsi, à la fois, comme un concept économique, avec une organisation de plus en plus territorialisée des processus productifs, épistémologique avec l'accroissement des travaux et des conceptualisations théoriques sous jacentes à ce nouveau paradigme (Khan, 2007) et comme un cadre pertinent pour la mise en place des politiques de développement durable.

L'objectif principal de cette contribution est de tenter de faire le point sur ce qui fait territoire. Nous traitons ce sujet sous l'angle économique et cherchons à montrer l'importance donnée à ce que nous appelons les deux piliers de la construction territoriale, à savoir la coordination des acteurs et les ressources territoriales. Un survey des différentes approches de l'économie territoriale permet de rendre compte à la fois, de l'importance de ces deux piliers dans la définition du territoire, et aussi de discuter de l'argumentaire développé par chacune des approches afin de les justifier. Un parti est donc pris pour une présentation ciblée sur deux notions fondamentales et communes à plusieurs approches territoriales, que sont la coordination et les ressources. Ces deux notions que nous qualifions de piliers sont primordiales pour comprendre le processus de construction et d'émergence de territoire.

\section{Le processus de construction territoriale}

Depuis trois décennies nous assistons à l'émergence d'un nouveau paradigme du développement qui participe à la réanimation de la science régionale, après quelques années de crise (Lacour, 1996). Le territoire est à juste titre considéré comme la " découverte " principale de la science régionale depuis les années 1980 (Samson, 2004). Il y aurait, selon Pecqueur (2006), un «moment territoire dans la régulation globale du système économique » résultant de la dialectique local/global. Au moment où la mondialisation donne un coup d'accélérateur aux délocalisations des firmes se localisant et se relocalisant en fonction des espaces qui offrent les coûts de production les moins coûteux, confortant l'idée d'un capitalisme mondial a-territorial. La mondialisation redonne également de la valeur au local avec ses spécificités et à l'ancrage territorial des entreprises. Nous sommes en présence de deux modèles de développement ancrés dans une double logique, d'un côté un système fordiste par nature a-spatial ayant pour moteur la grande firme nomade, en recherche permanente de coûts de production faible. De l'autre coté, un système territorial franchement opposé au premier, privilégiant l'ancrage spatial. Pour Guigou (1997), la mondialisation de l'économie entraine une territorialisation croissante des comportements. L'auteur avance cinq raisons majeures qui expliquent le retour du local: «la mobilité qui caractérise l'économie mondiale suscite, en retour, le besoin de sédentarité; l'éphémère engendre le besoin de repérer; l'homogène entraine le besoin de différences; la perte d'identité nationale suscite la montée en puissance des régionalismes; la mondialisation des marchés redonne une valeur accrue aux produits locaux;" (Guigou, 1997, p. 14)

5 Le territoire serait donc l'envers de la mondialisation, peu préoccupée par les contextes géographiques et culturels locaux. Mais avant de donner une définition de ce concept, il est intéressant de remonter tout d'abord à ses origines. Comme nous l'avons précédemment explicité, c'est grâce aux travaux d'Aydalot sur «le retournement des hiérarchies spatiales » et par la suite au groupe de recherche scientifique qu'il a crée en 1984 (le GREMI ; Groupement de Recherche Européen sur les Milieux Innovateurs), que les 
nouvelles dynamiques spatiales identifiées partout en Europe, mais surtout en Italie et en France, sont interprétées sous le filtre du territoire (Darchen et Tremblay, 2008). Au lieu de se focaliser sur l'entreprise, c'est le territoire qui retient l'attention. Le développement et l'innovation seraient secrétés non plus par les grandes entreprises, fer de lance du système fordiste, mais par le territoire et le milieu (Tabariès, 2005). Ce dernier « préexiste " à l'entreprise, c'est lui qui est à l'origine de l'entreprise et donc des dynamiques de développement et de croissance (Camagni, Maillat, 2006). L'approche par le territoire, à l'origine de l'économie territoriale actuelle en construction, a supplée les insuffisances des théories structuralistes (marxistes) et néoclassiques, qui étaient incapables d'expliquer les nouvelles dynamiques apparues dans les années 1970-80. Pour S. Darchen, D. G. Tremblay, (2008), Philippe Aydalot serait «le premier à considérer les structures socioéconomiques, leur dynamisme interne vis-à-vis du contexte extérieur ...» (p. 8) en théorie économique spatiale, même s'il "n'est pas le premier à intégrer une dimension spatiale dans l'analyse économique» (p. 8). Par son effort de conceptualisation, Aydalot arrive à réintégrer la dimension spatiale du développement via le concept de milieu. Le milieu est défini comme « un ensemble territorialisé dans lequel les interactions entre agents économiques se développent par l'apprentissage qu'ils font des relations multilatérales génératrices d'externalités spécifiques à l'innovation et par la convergence des apprentissages vers des formes de plus en plus performantes de gestion en commun des ressources" (Maillat, Quévit, Senn, 1993, p. 9). Le milieu est donc crée par l'interaction des agents et par les relations d'échanges continuelles qu'ils entretiennent. Ces interactions entre agents qui font face à des problèmes productifs et à des difficultés de coordination, entrainent une dynamique d'apprentissage, qu'ils mobilisent lors de la rencontre de nouvelles difficultés productives, sont donc de véritables ressources spécifiques latentes définies aussi comme des externalités territoriales, ancrées dans le territoire. Le milieu est considéré comme un " contexte favorable » à l'innovation et aux dynamiques de développement endogènes. Il permet de comprendre les trajectoires de développement de certaines régions. Le milieu innovateur s'articule autours de trois axes (Crevoisier, 2000) qui fondent sa structure : un axe technologique, qui considère l'innovation comme le résultat de l'articulation des ressources de la firme avec celles du territoire. Est analysé ici la capacité de la firme à " extraire une capacité technologique supplémentaire du milieu » - Un axe organisationnel, qui considère l'entreprise comme partie d'un ensemble territorial d'où émergent des relations avec d'autres acteurs du territoire comme les autres entreprises, et qui rentrent dans une logique de coopération/concurrence. La proximité fluidifie les relations de coordination entre les différents acteurs du milieu, elle est donc une source d'innovation, - Enfin, un axe territorial, qui renvoie aux différentes ressources du territoire pouvantêtre mobilisées pour l'innovation, comme le savoir faire, les compétences et le capital mais aussi la présence d'acteurs (entreprises, institutions...etc.).

6 Les recherches sur les milieux innovateurs initiées, comme on l'a souligné auparavant par Aydalot, et menées par le GREMI, s'inspirent fortement des théories évolutionnistes. A l'origine de celles-ci, l'ouvrage fondateur de Nelson et Winter en 1982. L'ambition de ces auteurs est selon Eric Brousseau (1999) l'étude des causes, des modes de diffusion et des effets du changement technique au sein d'une entreprise. La firme est décrite comme « un ensemble de compétences inscrites dans des routines évoluant sous l'effet d'apprentissages liés au processus de sélection » (Brousseau, 1999, p. 6) ; le but est de comprendre que l'innovation et la performance en général d'une organisation sont issues d'une dynamique d'apprentissage et de sélection résultant des interactions intra et extra firme. Pour les tenants de cette théorie, l'innovation est créée en dehors du marché, elle est secrétée par 
les coordinations hors marché des firmes qui mettent en commun leurs compétences ou leurs ressources. Pour Eric Brousseau (1999), cette théorie souffre d'une limite fondamentale qui est liée au fait que l'approche évolutionniste pense l'innovation en termes de coordination, mais n'explique pas comment celle-ci est construite entre les acteurs. La variable temporelle est fondamentale dans cette approche, c'est elle qui explique que l'innovation provient d'un mouvement long d'interaction entre les acteurs, à l'origine des processus d'apprentissages et de sélection qui génèrent les externalités positives nécessaires à l'innovation.

7 En parallèle, des travaux de même nature sont menés en Italie, essentiellement par Giaccomo Beccattini, Arnaldo Bagnasco, Carlo Trigilia et Sebastiano Brusco (1977 à 1988), sur les nouvelles dynamiques territoriales en Italie moyenne (Benko, Dunford, Lipietz, 1996). Entre le nord de l'Italie industrialisé et le sud sous développé, des villes et des vallées "qui par leurs seules forces, s'engageaient victorieusement sur le marché mondial à travers une industrie spécifique» (Benko, Dunford, Lipietz, 1996, p. 2). Les économistes italiens sont allés chercher un vieux concept «le district industriel » développé par Alfred Marshall, en partie oublié de la littérature économique, pour qualifier ces nouveaux territoires industriels. Il faut se rappeler avec Pecqueur (1996), qu'A. Marshall (1919) avait remarqué qu'au sein du mouvement dominant du début du siècle, s'appuyant sur les grandes firmes bénéficiant des économies d'échelle, il y avait «des anomalies » ou des exceptions, incarnées par une concentration de petites et moyennes entreprises très compétitives, fonctionnant selon le principe des économies externes, ou de ce qu'il appelait l'atmosphère industrielle et qu'il nomma districts industriels. Dans ce type d'organisation, la coordination se faisait par le marché et par le face à face avec une division sociale du travail entre des entreprises petites et moyennes spécialisées sur un segment de production. Le succès des districts italiens est attribué donc à cette atmosphère industrielle qui facilite les rapports de coordination marchande et non marchande entre les entreprises. Ces espaces industriels socialisés sont très vite assimilés à « des territoires construits par des acteurs» (Zimmermann, 2005) «mettant en valeur des ressources locales latentes » (Colletis et Pecqueur, 2004). Le territoire (district) tout comme le milieu dans la théorie des milieux innovateurs sont donc définis de la même façon, et renvoient à la même logique d'acteurs et de ressources. Ceci correspond en réalité à une évolution conceptuelle et à un rapprochement théorique des travaux menés par le GREMI sur les milieux innovateurs et ceux sur les systèmes productifs.

8 L'hypothèse fondamentale sous jacente à ces approches est que le territoire est un construit social résultant d'un processus de coordination des acteurs, réunis pour résoudre un problème productif inédit et de construction de ressources territoriales qui sont activées (ou révélées) pour une dynamique renouvelée du territoire.

9 Avant d'expliciter le contenu de chacun de ces deux piliers (fondamentaux) du territoire, il est nécessaire de citer l'ensemble des autres travaux qui ont suivis les premières conceptualisations de ce concept par Aydalot et Beccattini (1979). Au cours des années 1980-90 se développe en France les travaux sur les SPL (Systèmes Productifs Locaux) sous l'impulsion de Claude Courlet et de Bernard Pecqueur (Lipietz, 1995). Les districts et les milieux innovateurs sont considérés comme représentatifs de la nouvelle organisation industrielle qui fleurit partout dans le monde (pays développés et pays en voie de développement), quel que soit le milieu (rural ou urbain), qu'est le système productif local (SPL). La définition qui est donnée du SPL est celle d'une organisation territoriale de la production qui permet de mettre l'accent sur les autres facteurs non spécifiquement 
économiques qui participent à l'émergence de ces systèmes. C'est ainsi que «les rapports de collaboration entre les entreprises, les rapports entre le système productif et le système institutionnel, le savoir-faire et la mobilité des travailleurs dans l'agglomération de la production, le rôle des institutions sociales spécifiques qui interviennent pour appuyer le développement des entreprises » sont évoqués par Véronique Peyrache-Gadeau (2007, p. 3), comme essentiels à la compréhension des dynamiques à l'origine de ces SPL. Le SPL est vu comme un regroupement d'acteurs qui « confrontés à une même réalité technico-économique, développent collectivement et partagent des informations (scientifiques, techniques, industrielles, commerciales) sur les contraintes externes, sur les problèmes à résoudre ainsi que sur les modalités de solution possibles " (Peyrache-Gadeau, 2007, p. 3), cette organisation « s'appuie de surcroit sur des normes, implicites ou explicites, alliant règles du marché et code social. Ces normes et conventions, accords tacites, qui se sont instituées au fil du temps, répondent aux exigences particulières de la production sur lesquelles la culture professionnelle et technique locale s'est fondée » (ibid., 2007, p. 3). La sociologie industrielle montre que le principe commun à toutes les organisations territoriales est la coopération non spécifiquement marchande d'un réseau d'acteurs "encastré" dans des "réseaux sociaux» interprétés comme l'existence de "relations interindividuelles » fortes dans ces systèmes. Le partage d'un certain nombre de normes et de conventions provient pour le même auteur de l'existence de ces réseaux sociaux et des effets de proximité (Grossetti, 2004).

10 Les approches du territoire en termes de districts ou de SPL reprennent une grande partie de leurs concepts à l'économie institutionnelle ou encore à la nouvelle économie institutionnelle, qui s'inscrit dans les approches hétérodoxes de l'économie. Les porteurs de cette théorie (Williamson, 1975, à la suite des travaux de Coase, 1937, de Commons, 1934, et de Veblen, 1899) s'opposent à l'économie orthodoxe qui réduit l'espace à sa dimension coûts de transport, et l'homme à un homo-economicus rationnel dénué de toute dimension sociale et historique (Kherdjemil, 2000). Les agents économiques, assimilés ici à des homo-institutus, selon la terminologie de B. Kherdjemil (2000), vont devoir chercher un autre système de coordination que le marché, puisque ce dernier est source d'imperfections. De nouveaux modes de coordination ou « structures de gouvernance » sont alors « créés ». Le choix de la structure, selon Eric Brousseau (1997) d'après les travaux de Williamson (1975), se fera en fonction de celle qui minimise les coûts de transaction. Le choix de la structure de gouvernance dépend d'un processus d'apprentissage et "de sélection qui élimine les mécanismes de coordination inefficaces. Cette recherche se fait en fonction de deux éléments : les caractéristiques des transactions, d'une part, qui représentent la nature des difficultés de coordination; l'environnement institutionnel, d'autre part, qui désigne un ensemble de dispositifs collectifs qui constituent à la fois des contraintes et des points d'appuis pour la construction des structures de gouvernance.» (Brousseau, 1997, p. 9). L'action de l'individu dans l'espace est donc conditionnée par l'environnement institutionnel formel (le droit) et/ou informel (les normes et valeurs de chaque société) qui fixe les règles du jeu des mécanismes de coordination comme le contrat. Les acteurs, dans leur volonté de réduire l'incertitude et de minimisation des coûts de coordination, tiennent compte donc de deux variables: l'environnement institutionnel (formel et informel) et la psychologie des partenaires (confiance) (Kerdjemil, 2000). Cette démarche incite à penser le territoire comme construit collectif institutionnel ; c'est, selon Lahsen Abdelmalki, Daniel Dufourt, Thierry Kirat et Denis Requier-Desjardins, (1996), «la densité institutionnelle d'un espace» qui fait de lui un territoire. Appliquée au territoire, la perspective institutionnaliste le définit comme une création institutionnelle, c'est-à-dire " un espace dans lequel on observe non seulement "des procédures d'échanges non structurés et concurrentiels, des négociations et des 
relations contractuelles" mais aussi "l'ensemble des contraintes formelles sans lesquelles ces échanges et relations ne seraient pas possibles" " (Abdelmalki, Dufourt, Kirat, Desjardins, 1996, p. 5). Le territoire n'est plus perçu comme un contenant passif de facteurs de production, mais comme une création collective qui présente, selon L. Abdelmalki, D. Dufourt, T. Kirat et D.R-Desjardins, (1996), trois caractéristiques : «d'être l'émanation d'une logique de l'action collective; de conduire à une transformation des propriétés de l'espace banal; d'être assujetti à une exigence d'efficience schumpetérienne, c'est-à-dire de concourir à la mise en cuvre de procédures assurant la plus grande efficacité possible dans le processus de création de ressource.»(p.5)

11 Les approches régulationnistes nées de la fin des années 1970 et du début des années 1980 en France, grâce aux travaux de M. Aglietta et R. Boyer, dans une vision holiste, cherchaient à expliquer "comment les structures, malgré leur caractère contradictoire, se reproduisent-elles à travers les attentes, les intérêts, les actions à priori divergentes des agents économiques?» (Lipietz, 1995, p. 2). Tout comme la perspective institutionnelle, l'approche régulationniste avance que les formes institutionnelles et les habitudes sont celles qui « induisent ou contraignent les agents à se comporter de manière non antagonique à la reproduction de la structure.» (Ibid., 1995, p. 2). Ces institutions qui facilitent la résolution de conflits à l'intérieur d'une structure sont appelées "modes de régulation". Le mode de fonctionnement de ce système est le régime d'accumulation. A travers ces deux notions de mode de régulation et de régime d'accumulation, G. Benko (1996), M. Piore et Ch. Sabel (1984) vont interpréter l'émergence des nouvelles dynamiques territoriales comme la fin du système fordiste fondé sur un mode de régulation régit par la législation sociale, les conventions collectives et l'Etat providence et un régime d'accumulation fondé sur la redistribution des gains de productivité garantissant la croissance de la demande effective. Dans ce système la grande entreprise fait figure de prou et l'organisation de la production est très hiérarchique et rigide. La production de masse et standardisée crée une consommation de masse. Le district ou les SPL sont définis comme «la forme spatiale » du déploiement d'un nouveau mode de régulation postfordiste, fondé sur la flexibilité et une organisation horizontale de la production (Benko, 1996). Le territoire est donc l'organisation la plus efficace pour répondre aux nouvelles exigences des consommateurs, demandeurs de produits diversifiés, et à l'évolution rapide des processus de production liés aux nouvelles technologies.

12 Dans le même temps, l'école californienne de géographie économique d'Allen J. Scott, Michael Storper, et Richard Walker, développe des recherches de même nature dans le contexte métropolitain américain (Lipietz, 1995). Les métropoles sont structurées par les districts qui fructifient les économies d'échelle de variété. Toujours dans la lignée des travaux anglo-saxons, une large littérature s'est développée sous l'impulsion de M. Porter (2000) autour de la notion de cluster. Pour Pecqueur (2006), cette notion est affiliée aux travaux sur les districts italiens, elle est définie par la Harvard Business School comme " une concentration géographique de groupes d'entreprises interconnectées, d'universités et d'institutions associées, qui résulte des couplages (linkages) ou des externalités entre industries " (Samson, 2004). La définition du cluster, tout comme celle du district, des SPL ou des milieux innovateurs ont toutes pour point commun d'assimiler le territoire à un groupement d'acteurs spatialisés se coordonnant par des relations de marché et hors marché, valorisant les ressources locales pour résoudre un problème productif. Le territoire ne saurait se passer des ressources et des facteurs externes (Peyrache-Gadeau, 2007). D'un point de vue théorique, les travaux sur le territoire tentent de réintroduire 
l'espace en théorie économique en le considérant comme une construction sociale par des acteurs économiques mais aussi sociaux. Le territoire compte dans les dynamiques de développement. La mondialisation, en redonnant du sens au local, ouvre les portes aux approches hétérodoxes en économie pour fonder une nouvelle économie, socioéconomique comme celle à laquelle appelle $\mathrm{G}$. Benko et $\mathrm{A}$. Lipietz dans leur ouvrage "la richesse des régions, vers une nouvelle géographie socioéconomique ».

13 A l'intersection de l'économie industrielle et de l'économie spatiale, s'est développé un nouveau courant de pensée, dénommé "école de proximité » au cours des années 1990 en France. Ce rapprochement entre les deux disciplines est expliqué d'un côté, par le fait que les recherches en économie industrielle tiennent de plus en plus compte de la dimension spatiale, via l'ancrage des industries dans les territoires; de l'autre coté, les phénomènes industriels sont étudiés en économie spatiale. Ce courant s'oppose tout comme les approches précédemment présentées contre l'économie néoclassique (Gilly, Torre, 2000). L'approche par la proximité, géographique et organisationnelle, vise à dépasser la conception qui considère l'espace comme simple support des activités économiques pour l'enrichir et la compléter en privilégiant une lecture de l'espace en termes de coordination (Gilly et Torre, 2000). Il s'agit de comprendre comment les relations de coordination structurent l'espace. L'espace est perçu comme un construit, un lieu de création de ressource, ce qui l'éloigne de l'approche traditionnelle en termes de dotation de facteurs de production. Alors que la nouvelle géographie de Krugman (1991) postule l'existence d'externalités favorables à la concentration industrielle, les théoriciens de l'école de proximité tentent «d'ouvrir la boite noire » (Ibid., 2000) de ces externalités pour expliquer comment elles sont générées dans l'espace, la notion de proximité servant de grille de lecture. Le territoire est défini selon une double logique : la première l'inscrit dans un processus de valorisation des ressources selon une logique de proximité géographique; la seconde est celle qui lui permet de s'intégrer dans des circuits productifs externes par une proximité organisationnelle. Le territoire fait donc référence à deux types de proximité, la première géographique et la seconde organisationnelle. Cette dernière est subdivisée en deux autres sous-formes, selon la logique de similitude (proximité sociale) et selon la logique d'appartenance (proximité institutionnelle). Le territoire est un construit qui résulte d'une logique d'action collective qui mobilise les deux formes de proximité (Gilly, Torre, 2000). Sans proximité, il n'y a pas de coordination entre les acteurs, et sans celle-ci, il n'y a pas d'émergence de territoire. Elle est vue à la fois, comme une contrainte parce qu'elle limite l'action des agents à une communauté et à un espace donné, et un potentiel parce que quand elle est objectivée, elle conduit à des coordinations des acteurs, qui peuvent être ponctuelles et sporadiques ou régulières et répétées, générant des externalités positives, à la base de la construction territoriale.

\section{Premier pilier de la construction territoriale : la coordination des acteurs}

14 Cette partie entend expliciter le contenu du premier pilier de la construction territoriale. Elle explique à la fois ce qui est la coordination des acteurs, son importance dans le processus de construction de territoire et ses fondements épistémologiques et empiriques. Quelle que soit l'approche que l'on adopte ou l'optique dans laquelle on se situe, celle du milieu innovateur, du district, du SPL, du cluster ou de la proximité, le propos est le même : pour qu'un territoire émerge (se construise) il faut que les acteurs se 
coordonnent. Sans coordination, ou ce que Fabienne Leloup, Laurence Moyart et Bernard Pecqueur (2004), appellent "gouvernance locale", le territoire est voué à demeurer un espace passif qui subi les évolutions et les contraintes de son environnement extérieur. La régulation au niveau local correspond à deux évolutions majeures de la conception du développement.

Sur le plan empirique, elle correspond au constat établi à partir des années 1980 de l'échec de la régulation hiérarchique, centralisée. Les politiques publiques, venant « d'en haut » ne prennent pas en compte les particularités du local, qui est assimilé à un échelon de mise en application de la planification centralisée. Cette remise en cause de l'interventionnisme étatique fut accompagnée d'une prise de conscience par les acteurs locaux de la nécessité d'agir, et de participer à la gestion des affaires locales. Un processus de décentralisation est alors amorcé pour renforcer ces dynamiques locales endogènes. La gouvernance locale est donc née de la coïncidence de ces deux mouvements, émergence d'initiatives locales et désengagement progressif de l'Etat qui a accru, de ce fait, les prérogatives des acteurs locaux. On assiste alors du point de vue de l'Etat à un " mouvement de reterritorialisation de l'espace de l'action publique...enclenché, entre autre, par l'existence de nouveaux échelons d'intervention pour les politiques publiques et par une redéfinition des territoires de l'action publique (quartier, pays ou agglomération)...il ne s'agit plus de gouvernement d'unités administratives locales mais de gouvernance coordonnée des acteurs d'un territoire» (Leloup, Moyart, Pecqueur, 2004). La gouvernance n'est donc pas gouvernement, au sens d'une organisation politique qui administre un territoire, mais une coordination multiscalaire des acteurs privés et publics autour d'un projet en vu de résoudre un problème productif. Elle n'est pas, selon O. Crevoisier (2007) «imposée de l'extérieur» elle résulte "de l'interaction d'un certain nombre de groupes qui s'influencent mutuellement » (Crévoisier, 2007).

Sur le plan épistémologique, le concept de gouvernance ou de coordination des acteurs correspond à une volonté majeure de la part des théoriciens de l'économie spatiale " d'endogéneiser » la variable spatiale. Tenir compte de cette variable, revient à assimiler le territoire à un système de relations, qui par leurs interactions, aboutissent à la construction territoriale. Dans cette optique, le territoire est un construit socioéconomique résultant de la coordination des actions des différents acteurs du milieu. C'est ainsi que la théorie des milieux innovateurs fait de la variable organisation un axe fondamental du milieu. Olivier Crevoisier (2003) note que "les mécanismes de coordination sont au coeur des milieux innovateurs car ils articulent les aspects fonctionnels et territoriaux»; pour cet auteur "une entreprise, et en particulier une PME, n'est jamais qu'un élément inséré dans un système de production et dans un système territorial. Or, cette insertion passe par l'établissement de relations avec les autres entreprises de son environnement qui lui fournissent ses intrants ou lui achètent ses produits et services. Elle passe aussi par un ancrage territorial qui lui permet de mobiliser des ressources spécifiques et de participer aux réseaux locaux d'innovation et de soutien au système de production régional.» (p. 70). La mise en place de réseaux locaux de coordination et d'action est à l'origine de la création d'une frontière entre le milieu local, support de ces coordinations, et l'extérieur. Ces logiques aboutissent à la construction d'un «dedans", donc le territoire ou le milieu, par rapport à un "dehors ", soit l'environnement extérieur au milieu. Les formes de coordination décrites dans les milieux innovateurs sont de type concurrence/coopération. Dans le premier cas, la coordination se fait par le marché, le prix est la variable d'ajustement. Dans le second cas, la coordination se fait hors marché, elle repose dans ce cas sur des normes, des 
conventions formelles ou informelles qui minimisent les incertitudes et accroissent l'efficacité. La coordination hors marché aboutit, selon les théoriciens des milieux innovateurs, à la création d'un «capital relationnel » dans lequel, les acteurs du milieu se reconnaissent. Ces derniers partagent des valeurs (entrepreneuriales, familiales, professionnelles, etc.) qui sont à l'origine des relations de confiance et de réciprocité, source d'innovations.

Dans l'approche par les districts, Christian Longhi et Jacques Spindler (2000), à la suite de F. Vidal, (1998), affirment que les districts italiens sont nés "d'une innovation organisationnelle majeure portée par le territoire, la "déverticalisation" de la production dans les industries historiquement installées ». le district industriel est défini par Becattini (1992), comme " une entité socio-territoriale caractérisée par la présence active d'une communauté de personnes et d'une population d'entreprises dans un espace géographique et historique donné, (où existe) une osmose parfaite entre communauté locale et entreprise » (Longhi, Spindler, 2000, p. 10). C'est cette osmose, cette atmosphère caractérisée par une mise en synergie des forces locales, l'existence d'un climat favorable à la coopération et au travail collectif, qui est à l'origine du territoire. Tout comme le milieu innovateur, la nature de la coopération est également ici de type marchand et non marchand. La spécificité du cas des districts italiens et le fait que la plupart des entreprises sont issues de petites unités artisanales traditionnelles. La coordination est facilitée, d'autant plus ici, que les rapports entre les acteurs du milieu sont de type familial. La confiance est l'élément crucial de la coordination dans ce cas, elle repose sur le partage de valeurs et de normes communes, au bénéfice de la communauté. La mise en relation quotidienne des acteurs aboutie à des processus d'apprentissage qui facilitent l'adaptation aux mutations exogènes et à l'innovation. Alors que, dans le cas des districts italiens, « les relations interentreprises sont essentiellement fondées sur les rapports entre membres d'une communauté interagissant sur une base de confiance réciproque » (Longhi, Spindler, 2000), Dans le cas du district du BadeWurtemberg, "ces relations sont principalement sous-tendues par des organisations intermédiaires comme les associations d'entreprises, les syndicats, la chambre de commerce, les agences de transfert technologique, le gouvernement local ou les banques régionales » (p. 10). Les interactions sont donc de type institutionnel et formel. Ceci nous amène à qualifier les relations de coordination entre les acteurs comme un construit institutionnel, une sorte de mise en place d'une « bonne gouvernance » locale.

On se rend bien compte, que mis à part les contextes où les relations entre les acteurs sont de type communautaire ou familial, reposant sur une dimension historique, dans les autres cas la gouvernance est à construire, les mécanismes institutionnels pouvant jouer un rôle considérable. Dans les approches en termes de système de production localisé, G. Colletis et B. Pecqueur (2004), postulent le territoire comme un construit, celui-ci ne préexiste pas à l'action des acteurs, il est "révélé " par ces derniers. La question de la coordination est abordée "en dynamique ». En d'autres termes, celle-ci s'inscrit dans le temps, le présent est le résultat de l'action du passé. Le système territorial est « bâti sur la proximité géographique de ses acteurs, mais aussi sur la dynamique commune qui les rassemble " (Leloup, Moyart, Pecqueur, 2004); le construit territorial résultant «des relations, voire règles, normes et principes acceptés et mis en œuvre ensemble » (ibid., 2004). La coordination est toujours assimilée soit à une mobilisation des acteurs pour résoudre "un ou des problèmes productifs inédits ", soit "à un processus long de reconnaissance d'une identité ancrée dans la tradition, dans la coopération et en vue de réaliser un projet de développement collectif ". Les processus de coordination varient d'un espace à un autre et d'une période à une 
autre. Ils sont spécifiques, dans le sens où ils sont situés, relatifs à un contexte particulier. La gouvernance territoriale repose selon toujours les mêmes auteurs sur «le réseau et le flux ». Le réseau reflète une interconnexion entre les acteurs, qui peut être durable ou non, territoriale ou a-spatiale. Le flux renvoie au processus d'échange d'information et de bons procédés et dans ce cas, les éléments de proximité institutionnelle et géographique sont mobilisés pour délimiter le champ de ces flux et de ces réseaux.

Selon le type d'acteurs qui domine dans une structure de gouvernance, Leloup, L. Moyart, B. Pecqueur, (2004), à la suite de J.P. Gilly et J. Perrat (2003) définissent trois types théoriques de coordination: une coordination privée où l'acteur dominant est une organisation privée, une coordination institutionnelle ou publique, et une coordination mixte qui est un mélange des deux précédemment décrites. Les théoriciens de la proximité, quant à eux, axent pleinement leur raisonnement sur la coordination quand ils interrogent le territoire (B.olga, 2007). Pour eux la différenciation spatiale est fondée sur l'hypothèse de «non symétrie » et "non homogénéité» des «relations de coordination ». Les stratégies des acteurs sont à l'origine du territoire et de ses spécificités. Les relations de coordination interindividuelles et collectives sont appréhendées en termes de proximité. La proximité est modélisée comme utile mais non nécessairement comme condition à la coopération entre les acteurs. Les interactions de face à face facilitent le processus de création et de consolidation de liens hors marché essentiels à la construction territoriale. La proximité géographique est, dans le sens inverse, une contrainte pour les acteurs de territoires différents. Elle facilite l'émergence d'une proximité organisationnelle mais elle n'est pas, encore une fois, absolument nécessaire à un tel processus. La proximité organisationnelle est une proximité relationnelle, elle reflète "la capacité qu'offre une organisation de faire interagir ses membres" (Rallet, Torre, 2003). Ce type de proximité facilite la coordination entre les acteurs pour deux raisons. D'une part, la logique d'appartenance à une organisation induit inévitablement une interaction entre ses membres «fluidifiée » par l'existence de normes et de valeurs reconnus par tous. D'autre part, la logique de similitude qui prévaut dans une organisation accroit la coopération entre ses membres. Ces derniers sont réputés partager « un même système de représentation, de croyances et de symboles ». Le croisement des deux proximités est à l'origine des organisations territoriales qui ont émergé à partir des années 1970. C'est donc l'intensité des interactions des relations de proximité qui est à l'origine de la construction de territoire. La proximité qu'elle soit géographique ou organisationnelle nécessite d'être activée pour construire un espace collectif de coopération, générateur d'externalités positives (Gilly, Torre, 2000).

Lathéorie institutionnelle qui est celle qui traite en profondeur les logiques de coordination et de régulation entre les agents économiques a beaucoup inspiré les approches territoriales. Cette conceptualisation, qu'on a déjà définie ci-haut, provient des analyses renouvelées de l'entreprise par Coase en 1937. Celle-ci contrairement à la conception néoclassique n'est pas assimilée à une fonction de production qui intègre des inputs et produit des outputs. Tout comme le marché, le contrat et les conventions, l'entreprise ou la hiérarchie est modélisée comme un mode de régulation entre les acteurs à la recherche de coûts de transaction faibles. Pour remédier aux incertitudes et aux imperfections du marché, les agents économiques dotés d'une rationalité limitée recourent à d'autres types de régulation. La hiérarchie, les contrats et les conventions sont considérés comme plus efficaces que le marché dans l'allocation des facteurs de production. 


\section{Second pilier de la construction territoriale : la valorisation de la ressource}

21 Il est nécessaire dès à présent de noter que la conception de la ressource en économie des territoires, s'est complètement renouvelée à partir des années 1990, sous l'impulsion de l'économie territoriale, pensée en termes de construction de spécificités et d'ancrages (Peyrache-Gadeau, 2007). Le territoire comme ses ressources sont des construits sociaux (Khan, 2007) ; ils ne préexistent pas en amont à l'action des acteurs, ils sont créés ex-post par un processus de raisonnement et de tâtonnements itératifs. Mais avant d'aborder la définition actuelle, il convient de présenter la conception traditionnelle telle qu'elle a été énoncée par les différentes disciplines qui se sont intéressées à cette notion.

Longtemps, le cloisonnement entre géographie physique et géographie humaine a réduit la définition de la ressource à sa dimension naturelle. Pour Vergnolle Mainar (2006) ces ressources naturelles sont « considérées comme source de richesses et sont alors abordées sous l'angle de leur exploitation et des activités qu'elles permettent» (p. 3), celles-ci ont nourri un discours déterministe qui soutenait que la richesse d'un territoire se mesurait à l'étendue des ressources de son sol et sous-sol. Plus le territoire est vaste, plus les richesses sont importantes et variées et plus le territoire est considéré comme puissant. Pour Jacques Lévy et M Lussault (Lévy, Lussault, 2003) la ressource est "une réalité entrant dans un processus de production et incorporée dans le résultat de celui-ci», soit un moyen qui sert à satisfaire les besoins et à créer de la richesse. Traditionnellement les ressources naturelles sont celles qui sont utilisées pour illustrer cette définition (Glon, 2007). Sont différenciées, à partir de processus biophysiques, celles qui sont renouvelables comme la forêt et celles qui ne le sont pas comme les ressources minières. Et encore, la surexploitation actuelle de certaines ressources peut remettre en cause le caractère renouvelable ou non d'une ressource (ressource halieutique par exemple).

23 En sciences économiques et sociales, les ressources sont celles qui ont un prix sur le marché et leur importance est étroitement corrélée à leur valeur. Selon les différents courants de pensée, on retient le travail, le capital et les matières premières comme les ressources principales du territoire. De nos jours, les ressources cognitives sont additionnées aux précédentes au regard de la nouvelle économie (économie de la connaissance). Peu à peu, la conception de la ressource s'élargit, le caractère marchand n'est plus le seul critère à être retenu pour la définir, de nouveaux objets sont désormais qualifiés de ressources, comme le paysage, le climat, la forêt, l'eau. Ces biens collectifs sans valeur marchande apparente retiennent l'attention des acteurs, en concomitance avec les nouvelles préoccupations de la société. De nouvelles fonctions leurs sont attribuées, le paysage devient par exemple une ressource mobilisée par les territoires sensibles comme un facteur de différenciation et d'attractivité auprès des touristes. Ce changement de cap s'explique pour plusieurs raisons, à la fois d'ordre économique, social et environnemental. Sur le plan économique, Pecqueur note que le passage de la période fordiste (Lipietz, 1995), à la période post fordiste, ouvre des voies de développement insoupçonnables jusque-là pour les territoires fragiles victimes du système fordiste. Les handicaps traditionnellement perçus comme tels dans les territoires ruraux et de montagne, comme le relief, la pente, la rudesse de la vie, l'archaïsme de la société rurale et le climat difficile deviennent à l'aune du nouveau système des ressources potentielles, 
convenablement valorisées; elles sont une source de dynamisme, de richesse et d'une rente territoriale de situation (Mollard, Pecqueur, Moalla, 2001).

Aux côtés du système fordiste de production de masse et standardisée, régulé par le marché et les prix, dominé par les grandes structures qui laissent très peu de chance aux petits concurrents, il existe un autre système, dont les nombreux qualificatifs qui lui sont attribués (après fordisme, post-fordisme, accumulation flexible...) témoignent de la difficulté qui subsiste encore à le qualifier. Celui-ci s'articule autour du couple différenciation-spécification. Ce système échappe à la concurrence traditionnelle par les prix et les coûts, les nouvelles formes de concurrence passent par la différenciation de l'offre et sur les signaux de qualité (Mollard, Pecqueur, Moalla, 2001). Tous les objets du territoire acquièrent de nouvelles valeurs, les perceptions changent et tout devient une ressource potentielle, et un élément de différenciation. La conception de la ressource passe ainsi d'un stock de matières disponibles sur le territoire à celle d'une construction par les acteurs qui qualifient et requalifient l'ensemble des objets du territoire. Cette qualification est étroitement associée aux valeurs que les acteurs souhaitent leurs attribuer, elles mêmes dépendante des évolutions sociales et culturelles de la société globale. La ressource n'est plus un simple intrant (stock de matière) mobilisé dans les processus productifs, mais bien un résultat d'une mobilisation, d'une concertation et d'une coordination des acteurs autour d'un objet, pas nécessairement matériel, qui peut être une histoire, un mythe, une identité ou une valeur commune.

Sur le plan social, face au système englobant qui véhicule des valeurs dans lesquelles les sociétés locales ne se retrouvent pas, les identités locales sont objectivées et transformées en ressources spécifiques. Le local s'affirme mais dans un esprit d'ouverture en faisant valoir à la fois ses potentialités endogènes et sa capacité à allier celles-ci avec les ressources exogènes. Autre fait social, la demande des consommateurs a changé, ces derniers cherchent à se différencier par leurs goûts et leurs besoins, ils réclament la différence et changent rapidement leurs préférences. Ils sont sensibilisés sur les dangers d'un système productiviste soucieux simplement de rendement et de profit. Les besoins en sécurité alimentaire sont allés crescendo, et les produits de qualité et/ou labellisés connaissent une demande croissante. Les consommateurs réclament également la traçabilité et l'authenticité des produits et souhaitent consommer à travers eux une culture, un territoire. Ces bouleversements sociaux sont ainsi à l'origine de la reconsidération du territoire et des ressources qui lui sont associées.

Sur le plan environnemental, de nouvelles préoccupations ont émergé, le développement n'est plus une question purement économique et marchande, il est aussi synonyme d'équité sociale, de préservation de la nature et des ressources. La nouvelle perception de la notion de ressource se soucie à la fois de l'exploitation raisonnable de celle-ci et de sa durabilité. Les acteurs du territoire sont appelés à revoir leur conception de la ressource dans un souci de durabilité (Peyrache, 2007).

Il apparait ainsi que la ressource qualifiée ici de territoriale en ce sens qu'elle est endogène à celui-ci, constitue le deuxième pilier de la nouvelle économie territoriale en construction. Pour Pecqueur, l'originalité de ce modèle est « de remonter à l'amont de l'actif pour considérer toutes les ressources comprenant notamment celles qui ne sont que virtuelles n'ayant pas d'existence préalable à l'action de les faire naître» (Pecqueur, 2006, p. 9), à l'état virtuel tout peut constituer une ressource, le passage de l'état virtuel à l'état d'actif suppose une intentionnalité des acteurs qui se mobilisent pour révéler et construire l'actif. La ressource n'est donc pas un actif, elle ne le devient qu'après un processus 
d'activation. Plusieurs typologies de la ressource sont présentées par les chercheurs, nous les détaillerons dans la suite, en corollaire, à chaque type de ressource correspond un mode d'activation que nous développerons également ci-après.

La notion de ressource est relative (Corrado, 2007), pour Vergnolle Mainar (2006) à la suite des travaux de Brunet, Ferras et Théry (1992), celle-ci n'existe que révélée, nantie d'une valeur d'usage. C'est donc «la valeur qu'une société ou un individu attribue à tel ou tel élément qui conduit à le considérer comme ressource » (p.4), les ressources sont donc toujours « inventées, parfois bien après avoir été découvertes » (p. 4), la ressource est donc relative au contexte spatial et temporel qui l'a vu naitre. Ce qui est une ressource à un moment donné et dans un territoire donné, ne l'est pas forcement à un autre moment et dans d'autres lieux. A titre d'exemple, les éléments de fragilité (Relief, climat, paysage...etc.) des territoires de moyenne montagne sont revus de nos jours sous un angle positif. La demande croissante de la société en termes de consommation de ces espaces affecte de nouvelles valeurs à ces territoires, les mêmes objets (handicaps) sont transformés en ressources (paysage, image...etc.). Les ressources territoriales, pour reprendre l'expression de Pecqueur sont «clouées » au lieu. Les spécificités locales, la culture locale et l'esprit du lieu expliquent l'ancrage des ressources au territoire.

Pour Glon (2007), l'émergence d'une approche des ressources est intimement liée à " l'ampleur et à l'implication de socio-systèmes", dont l'action ou le projet "s'inscrit dans une propension plus ou moins forte à interroger la mémoire des lieux ou d'une collectivité, des savoirfaire qui s'y manifestent, des expériences qu'elle a traversées et connaît » (p. 1); l'importance de la proximité et du maillage y est soulignée. Le fondement même du territoire ne saurait exister sans une dose de proximité qu'elle soit géographique, institutionnelle ou organisationnelle (J.P. Gilly, A. Torre, 2000).

Pour Camagni, Maillat et. Matteaccioli (2004) du GREMI, la ressource est secrétée par un milieu innovateur, ils insistent sur le rôle des réseaux d'innovation et des effets d'apprentissage dans le processus de révélation de la ressource. Pecqueur (2004) note que l'ensemble des travaux actuels sur le territoire raisonnent en termes de création de ressource et de production d'externalités, via la coordination. Les seules ressources dont le territoire dispose sont «liées à une trace de coordination passée (mémoire, confiance) et à un potentiel, une latence, ou encore une virtualité de nature cognitive qui demandent qu'à être activées ou révélée à la faveur d'un problème productif» (Colletis, Pecqueur, 2004, p. 10); certains auteurs utilisent d'autres qualificatifs pour désigner ces ressources (institutions) tels que le capital social ou le patrimoine. Pour Colletis et Pecqueur (2004), ces deux notions renvoient à la même définition qui est donnée à la ressource, ils suggèrent d'intégrer ces deux notions avec celle de la ressource dans une "théorie générale des ressources".

31 En étudiant comment se construit un système culturel localisé dans les stations touristiques de nature, J. Corneloup (2004) montre que la réussite de certaines stations de sport d'hiver qui ne fonctionnent pourtant pas « selon le modèle de rationalité parfaite et $d u$ management total », est liée " au capital culturel que possède la station en tant que ressource fondamentale pour le bon fonctionnement de l'économie territoriale» (p. 13); celle-ci se construit sur les spécificités culturelles locales. La bonne gouvernance ou encore la qualité d'un site ne se mesure pas seulement «à la qualité des services, du prix de l'immobilier, des spécificités environnementales du site » (ibid., 2004, p. 14), mais aussi, sur « des liens, des pratiques sociales qui émergent localement ", les touristes aiment se retrouver 
sur un site «entre des gens partageant la même vision du monde et ayant des affinités communes »(ibid., 2004, p. 14).

32 La ressource suit un cycle de vie en deux étapes: la genèse ou l'identification et la valorisation qui peut prendre plusieurs formes. Une ressource se déploie, se transforme quelquefois régresse pour redevenir un potentiel latent qui n'attend qu'à être réactivé par de nouvelles valeurs ou de nouveaux usages que la société décide de lui assigner. Quatre caractéristiques fondamentales sont attribuées à la ressource (Gumuchian, Pecqueur, 2007) : la position; où se trouve-t-elle sur le territoire ? Endogène ou exogène? La constructibilité ; comment la ressource naît (stratégies d'acteurs) ? Et comment s'intègre-elle dans un processus de mise en valeur? La complexité systémique ; comment elle se combine avec d'autres ressources sur le territoire? Le sens et la temporalité ; quelle est sa nature: matérielle ou idéelle? Et son cycle de vie (sa durabilité) ? Les ressources tiennent une place fondamentale dans le processus de construction de territoire. Leur révélation, valorisation et spécification par les coordinations des acteurs conditionne l'émergence des territoires.

\section{Conclusion}

Dans cet article nous avons questionné le contenu de la construction territoriale au travers une grille de lecture reprenant les différentes approches de l'économie territoriale. Notre propos consiste à mettre l'accent sur deux notions qui nous semblent fondamentales à la compréhension du territoire. Il est clair que ce concept souvent mobilisé par les chercheurs est complexe et multidimensionnel. Il existe par ailleurs une multitude d'autres approches qui n'ont pas été abordées dans ce travail. C'est le cas notamment de l'approche géographique, sociale et politique du territoire. Nous n'avons pas, non plus, tenté de donner une définition complète et exhaustive de ce que recouvre ce concept. La complexité d'une telle tâche dépasse le cadre de cet article. Notre objectif consistait à reprendre deux notions, communes à l'approche économique et qui nous semblent fondamentales à la compréhension du processus de construction territoriale, afin de préciser leurs contenus, de discuter leurs définitions et leurs fondements théoriques.

Le territoire ne saurait se construire sans une mobilisation des acteurs et sans une mise en œuvre des ressources territoriales. Ce sont ces deux piliers de la construction territoriale que nous avons voulu mettre en perspective. La coordination des acteurs relève d'une prise de conscience de la nécessité de mise en commun des forces et des intérêts individuels au profit du bien de la communauté. Elle est indispensable à la mise en place de dispositifs de gouvernance. Les ressources territoriales construites à la suite d'une mobilisation des acteurs sont en fin de compte la véritable richesse qui permet au territoire de se développer. Une bonne gouvernance ne saurait suffire à la construction territoriale sans les ressources territoriales. Inversement, les ressources territoriales ne sauraient apporter la prospérité à un territoire sans une bonne gouvernance et donc coordination des acteurs. Les ressources territoriales et la coordination des acteurs sont ainsi les deux piliers indispensables à la construction de territoire. 


\section{BIBLIOGRAPHIE}

Abdelmalki L., Dufourt D., Kirat T., Requier-Desjardins D., 1996. Technologie, institutions et territoires : le territoire comme création collective et ressource institutionnelle, in Bernard Pecqueur, Dynamiques territoriales et mutations économiques, éditions l'Harmattan.

Aydalot Ph, 1986. Milieux innovateurs en Europe, (éd.), GREMI.

Aydalot Ph., 1980. Dynamique spatiale et développement inégal, Economica, Paris.

Beccattini G., 1989. Les districts industriels en Italie, in Maruani M. et al. (éd.), La flexibilité en Italie, Syros, Paris, p. 261-268.

Benko G., Lipietz A., 2000. La richesse des régions, la nouvelle géographie socio-économique, Ed. Economica.

Benko G., 1996, « Géographie économique et théorie de la régulation », Finisterra, XXXI, 62, 1997, pp. 7-28.

Benko G, Dunford M., Lipietz L., 1996. Les districts industriels revisités ", in B. Pecqueur éd. Dynamiques territoriales et mutations économiques, L'Harmattan, 1996.

BrousseauE., 1997, « Néo-institutionnalisme et Évolutionnisme: Quelles Convergences ?», Economies et Sociétés, HS 35, N¹,1/1999,

Bouba Olga O., 2007. Un approfondissement du modèle exit-voice par l'économie de proximité, CRIEF-TEIR, EA 2249, Université de Poitiers.

Callois., 2004, «Capital social et développement économique local : un test économétrique sur l'espace rural français », XLème Colloque de l'ASRDLF, Convergence et disparités régionales au sein de l'espace européen, Les politiques régionales à l'épreuve des faits.

Camagni R., Maillat D., 2006. Milieux innovateurs, théorie et politique, Ed. Economica.

Camagni R., Maillat D., Matteaccioli A., 2004. Ressources naturelles et culturelles, milieux et développement local, Neuchâtel, institut de recherches économiques et régionales, éditions EDES.

CERAMAC, 1999. Moyennes montagnes européennes, actes du colloque de Clermont-Ferrand

Colletis-Wahl, Corpataux K., Crevoisier J., O, Kebir, L., Pequeur, B. et Peyrache-Gadeau, V., 2006. L'économie territoriale Une approche générale pour mieux comprendre et faire face à la globalisation, GRET.

Corrado F., 2007. Le traitement des ressources dans la politique du développement local : le cas de la ville de CASERTA, in Les dynamiques territoriales débats et enjeux entre les différentes approches pluridisciplinaire, XLIIIè colloque de l'ASRDLF, Grenoble-Chambéry, 11, 12, 13 juillet 2007.

Corneloup J., 2004. Le marquage culturel des territoires touristiques de nature, in première rencontre Franco-Espagnoles de tourisme, Arles 9 et 10 décembre 2004.

Crevoisier O., 2003, «Economie, Territoire et Durabilité : une approche par les milieux innovateurs ", in Ruegg J., Géographie et Développement Durable, Presses Polytechniques Romandes.

Crevoisier O., Kebir L., 2007. Les ressources culturelles entre réseaux internationaux et développement local, Working paper 6/2007-F, GRET. 
Crevoisier O., Camagni R., 2000. Les milieux urbains : innovation, systèmes de production et ancrage, éditions EDES.

Darchen D., Tremblay G., 2008. « Les milieux innovateurs et la classe créative : revue des écrits et analyse de leur application en milieu urbain », Note de recherche de la Chaire de recherche du Canada sur les enjeux socio-organisationnels de l'économie du savoir.

Joye J-F., 2007. Organiser les dynamiques territoriales : le rôle de la ressource juridique, analyse de solutions : collectivité chef de file et contrat Etat-Région, in Les dynamiques territoriales débats et enjeux entre les différentes approches pluridisciplinaires, XLIIIè colloque de l'ASRDLF, GrenobleChambéry, 11, 12, 13 juillet 2007.

Gilly J-P., Torre A., 2000. Dynamique de proximité, Ed. L'Harmattan, collection Emploi, industrie et territoire.

Glon E., 2007. Les ressources territoriales, une notion clé pour une meilleure re-connaissance des territoires dans le global, in Les dynamiques territoriales débats et enjeux entre les différentes approches pluridisciplinaire, XLIIIè colloque de l'ASRDLF, Grenoble-Chambéry, 11, 12, 13 juillet 2007.

Greffe X., 2002. Le développement local, Bibliothèque des territoires, Ed. de l'aube DATAR, Paris.

Grossetti, M., 2001a. « Les effets de proximité spatiale dans les relations entre organisations : une question d'encastrements », Espaces \& Sociétés 101-102, 203-219.

Grossetti M., 2004. «Concentration d'entreprises et innovation : Esquisse d'une typologie des systèmes productifs locaux ", Géographie, économie, société, 2004, Vol. 6, n².

Guigou J-L, 1997, « Le paradoxe : Mondialisation-Territorialisation », in Guy Loinger et Jean-Claude Némery, 1997, « Construire la dynamique des territoires... Acteurs, institutions, citoyenneté active », Ed. L'Harmattan, collection administration et aménagement du territoire.

Gumuchian H., Pecqueur B., 2007. La ressource territoriale, Ed. Economica.

Kahn R., 2007. « Une approche pluridisciplinaire de la dimension culturelle du développement territorial ", in Les dynamiques territoriales débats et enjeux entre les différentes approches pluridisciplinaire, XLIIIè colloque de l'ASRDLF, Grenoble-Chambéry, 11, 12, 13 juillet 2007.

Kherdjemil B., 2000. La notion d'espace institutionnel, Classification JEL : K11, K12, L22, R10, R15.

Lacour C., 1996. « La tectonique des territoires: d'une métaphore à une théorisation ", in Pecqueur B., (ed.) Dynamiques territoriales et mutations économiques, L'Harmattan, Paris.

Landel P.-A., Pecqueur B., 2005. « La culture comme ressource territoriale spécifique », Communication présentée au colloque de l'Association de Science Régionale de langue Française, Bruxelles, 1-3 septembre 2004, $16 \mathrm{p}$.

Leloup F., Moyart L., Pecqueur B., 2004. « La gouvernance territoriale comme nouveau mode de coordination territoriale? ", $4^{\text {ème }}$ journées de la proximité.

Le Moigne J-L., 1994. la théorie du système général, théorie de la modélisation, collection Les Classiques du réseau intelligence de la complexité

Lévy J., Lussault M., 2003. Dictionnaire de la géographie et de l'espace des sociétés, Ed. Belin.

Lipietz A., 1995. De la régulation des espaces aux espaces de régulation, in Boyer\&Saillard, L'état de la théorie de la régulation, Ed. la découverte, paris.

Maillat D., Perrin J-C., 1992. Entreprises innovatrices et développement territorial, GREMI/EDES 1992. 
Maillat, Quévit et Senn (éds), 1993. Réseaux d’innovation et milieux innovateurs : un pari pour le développement régional, GREMI/EDES 1993.

Maillat, Crevoisier, Lecoq, 1993. Réseaux d'innovation et dynamique territoriale : le cas de l'Arc jurassien, in Denis Maillat, Michel Quévit, Lanfranco Senn (éds), 1993, Réseaux d'innovation et milieux innovateurs : un pari pour le développement régional, GREMI/EDES 1993.

Maillat, 1992. La relation des entreprises innovatrices avec leur milieu, in Denis Maillat et JeanClaude Perrin (éds), 1992, Entreprises innovatrices et développement territorial, GREMI/EDES 1992.

Marshall, A., 1890. Principes d'économie politique (traduction française 1906).

Mollard A., Pecqueur B., Moalla M., 2001. Offre de produits et services territorialisés et approche lancastérienne de la demande de biens combinés, the third congress on proximity "new growth and territories ", paris dec. 13-14th 2001, university of paris south\&institut national de la recherche agronomique.

Moine A., 2005. Le territoire comme un système complexe, des outils pour l'aménagement et la géographie, ThéMA UMR 6049 CNRS - Université de de Franche-Comté,

Pecqueur B., 1996. Dynamiques territoriales et mutations économiques, Ed. L'harmattan.

Pecqueur B., 2004. Vers une géographie économique et culturelle autour de la notion de territoire, Géographie et Cultures, numéro spécial, pp. 22-37.

Pecqueur B., Colletis G., 2004. Révélation de ressources spécifiques et coordination située, Colloque international sur "l'économie de proximité ", Marseille, 8-9 juin 2004, Revue Economie et Institution.

Pecqueur, B., et Zimmermann, J.-B., 2004. Economie de proximités, Hermès.

Pecqueur B., 2006. Le tournant territorial de l'économie globale, revue Espace et Société, $\mathrm{n}^{\circ}$ 124-125 2006.

Peyrache-Gadeau V. 2007. Modes de développement et vulnérabilités : quels enjeux pour l'économie territoriale?, in Les dynamiques territoriales débats et enjeux entre les différentes approches pluridisciplinaire, XLIIIè colloque de l'ASRDLF, Grenoble-Chambéry, 11, 12, 13 juillet 2007.

Peyrache-Gadeau V. 1999. « La contribution de Philippe Aydalot à

l'édification de la théorie des milieux innovateurs ", Revue d'Economie Régionale et Urbaine $\mathrm{n}^{\circ}$ 3, pp. 617-632.

Rallet A., Torre A., 2007. La proximité à l'épreuve des technologies de communication, Ed. L'Harmattan. Rieutort L., 2005. Diagnostic préalable à la mise en place d'un salon de la moyenne montagne, rapport final de synthèse, foire exposition Clermont-Cournon, 2005.

Samaganova A., Ivan Samson, 2007. Typologies de ressources et ouverture des territoires, in Les dynamiques territoriales débats et enjeux entre les différentes approches pluridisciplinaire, XLIIIè colloque de l'ASRDLF, Grenoble-Chambéry, 11, 12, 13 juillet 2007.

Samaganova A., 2007. La culture comme ressource territoriale en économie de la connaissance, in Les dynamiques territoriales débats et enjeux entre les différentes approches pluridisciplinaire, XLIIIè colloque de l'ASRDLF, Grenoble-Chambéry, 11, 12, 13 juillet 2007.

Samson I., 2004. Territoire et système économique, communication aux 4èmes journées de la proximité : Proximité, Réseaux et Coopération, Marseille les 17 et 18 Juin 2004. 
Tabariés M., 2005. Les apports du GREMI à l'analyse territoriale de l'innovation ou 20 ans de recherche sur les milieux innovateurs, Matisse, Cahiers de la MSH, Centre National de la Recherche Scientifique.

Vergnolle Mainar C., 2006. Géographie et EEDD : la notion de ressource, à la croisée des approches disciplinaires et interdisciplinaires, colloque international des journées d'études de didactique de l'histoire et de la géographie, Reims, 23-24 octobre 2006.

\section{RÉSUMÉS}

L'objectif principal de cet article est de tenter de faire ressortir les composantes principales de ce qui fait territoire. Dans la foisonnante littérature qui s'est développée depuis trois décennies sur cette catégorie conceptuelle, il est difficile de s'y retrouver. D'où la nécessité de tenter une synthèse qui ne prétend nullement à l'exhaustivité, mais qui simplement essaie de faciliter la lecture de ces travaux. L'hypothèse fondamentale sous jacente aux différentes conceptions du territoire est que le territoire est un construit social résultant de la combinaison d'une coordination d'acteurs, non résolument économique, réunis pour résoudre un problème productif inédit et de ressources territoriales qui sont activées pour une dynamique renouvelée du territoire. Ce dernier est donc caractérisé par la mobilisation des acteurs, qui rentrent dans une logique de coopération et de coordination de leurs actions. Ces logiques de coopération peuvent se concrétiser par la mise en place de systèmes de gouvernance variés, différents du mode de régulation traditionnel qu'est le marché. Des contrats, des conventions, des réseaux et une coopération formelle et/ou informelle sont la panoplie des modes de régulation qui sont mobilisés par les acteurs pour faire émerger leur espace en tant que territoire dynamique. En deuxième lieu, le territoire repose sur la révélation de ressources latentes, de préférence spécifiques, non transférables, identifiées et activées par la mobilisation des acteurs.

The main objective of this article is to try to highlight the main components of what makes territory. In the abundant literature developed over the past three decades on this conceptual category, it is difficult to find an appropriate definition. Hence the need to attempt a synthesis which does not pretend to be exhaustive, but simply trying to facilitate reading of this new paradigm of development is necessary. The basic assumption underlying the different conceptions of the territory consider it as social construct derived from a combination of coordination of actors, meeting to solve a productive problem and resources which are activated for a renewed dynamic of the territory. The latter is characterized by the mobilization of actors who following a logic of cooperation and coordination of their actions. The logic of cooperation can be realized through the establishment of diverse governance systems, different from the traditional mode of regulation which is the market. Contracts, agreements, networks, and formal or informal cooperation are a range regulation models that are mobilized by the actors to transform their space in a dynamic territory. Secondly, the territory is based on the revelation of latent resources, preferably specific, non-transferable, identified and activated by the mobilization of actors.

\section{INDEX}

Mots-clés : territoire, construction territoriale, coordination des acteurs, ressources territoriales

Keywords : territory, territorial development, coordination of actors, territorial resources 


\section{AUTEUR}

\section{HADJOU LAMARA}

Hadjou Lamara est Doctorant à l'Université Blaise Pascal et l'Université de Thessalie, CERAMAC, Maison des Sciences de l'Homme, 4, rue Ledru, 63057 Clermont-Ferrand. Tel : 0628157824 lhadjou [at]yahoo.fr 